\title{
A SPECIFIC FORM OF GROTHENDIECK'S INEQUALITY FOR THE TWO-DIMENSIONAL CASE, WITH APPLICATIONS TO C*-ALGEBRAS
}

\author{
by G. J. O. JAMESON
}

(Received 25th March 1993)

\begin{abstract}
We characterize bilinear forms $V$ on $l_{x}^{2}$ such that $V(e, e)=\|V\|=1$ in terms of their matrices. For such $V$, we prove that $|V(x, y)|^{2} \leqq \phi\left(|x|^{2}\right) \psi\left(|y|^{2}\right)$ for all $x, y$, where $\phi(x)=V(x, e), \psi(y)=V(e, y)$. Some other properties of such forms are given.
\end{abstract}

1991 Mathematics subject classification: 46L30.

\section{Introduction}

Let $A, B$ be complex $C^{*}$-algebras with identities $e_{A}, e_{B}$. We say that a bilinear form $V$ on $A \times B$ is unital if

$$
V\left(e_{A}, e_{B}\right)=\|V\|=1
$$

Note that if $A, B$ are finite-dimensional and $V$ is any bilinear form with $\|V\|=1$, then there will be unitary elements $x_{0} \in A, y_{0} \in B$ such that $V\left(x_{0}, y_{0}\right)=1$, and a unital form $V_{1}$ is then obtained by putting $V_{1}(x, y)=V\left(x_{0} x, y_{0} y\right)$.

If $V$ is unital, then states $\phi$ (on $A$ ), $\psi$ (on $B$ ) are defined by: $\phi(x)=V\left(x, e_{B}\right)$, $\psi(y)=V\left(e_{A}, y\right)$. A result of Haagerup [2] states that we then have

$$
|\operatorname{Re} V(x, y)|^{2} \leqq \phi\left(x^{2}\right) \psi\left(y^{2}\right)
$$

for self-adjoint $x, y$. This is not true for $|V(x, y)|$ or for non-self-adjoint elements, though Grothendieck-type theorems assert the existence of other states $\phi^{\prime}, \psi^{\prime}$ giving such inequalities with an intervening constant.

This result is an essential lemma in Haagerup's proof of the $C^{*}$-algebra version of Grothendieck's inequality. A second essential, and rather tricky, lemma (Lemma 3.2) deals with $\operatorname{Im} V(x, y)$ in the very special case $A=B=l_{\infty}^{2}$. Bilinear forms on $l_{\infty}^{2}$ are thus of key importance for the general case. They are also of considerable interest in their own right. Our main theorem (Theorem 2) states that for a unital bilinear form $V$ on $l_{\infty}^{2}$, with $\phi, \psi$ defined as above, we have

$$
|V(x, y)|^{2} \leqq \phi\left(|x|^{2}\right) \psi\left(|y|^{2}\right)
$$


for all $x, y$. This easily implies Haagerup's Lemma 3.2. Furthermore, it reproves the known fact [1], [7] that Grothendieck's theorem holds with constant 1 for $l_{\infty}^{2}$, but with the extra feature that it identifies the dominating states as the $\phi, \psi$ defined above. There would appear to be no other known situation where there is a ready-made formula for the "best" dominating states for a given bilinear form. We show by an example that the $\phi, \psi$ above (which are, of course, the natural candidates) are no longer the best choice even in the case $l_{\infty}^{2} \times l_{\infty}^{3}$; the best constant is, of course, already unknown for this case. Hence it is not surprising that some quite delicate work is needed to obtain our Theorem 2.

It is not at all easy, a priori, to recognise when a bilinear form on $l_{\infty}^{2}$ is unital. Our Theorem 1 provides a full-and perhaps unexpected - characterization of such forms in terms of the real and imaginary parts in the $2 \times 2$ matrix $\left(V\left(e_{j}, e_{k}\right)\right)$ : in the notation used below, the non-trivial condition involved is $h^{2} \leqq b c d+a c d+a b d+a b c$. The deduction of Theorem 2 then roughly mimics one of the standard proofs of the commutative Grothendieck inequality; in a quite natural way, the inequality just mentioned is seen to be exactly what is needed. On the way to Theorem 1, we find further interesting properties of unital forms, in some cases applying to $C^{*}$-algebras generally. For example, if $0 \leqq x \leqq e_{A}$ and $0 \leqq y \leqq e_{B}$, then the least possible value for $\operatorname{Re} V(x, y)$ is exactly $-\frac{1}{8}$.

We finish by giving short proofs, avoiding our theorems 1 and 2, of Haagerup's Lemma 3.2 and of the fact that the complex $2 \times 2$ Grothendieck constant is 1 .

\section{Elementary results}

Notation. As usual, $l_{\infty}^{n}$ denotes $\mathbb{C}^{n}$ with supremum norm. The $j$ th unit vector is denoted by $e_{j}$ and $e_{1}+\cdots+e_{n}$ by $e$. For $x, y$ in $l_{\infty}^{n}$, the elements $|x|$ and $x y$ are defined pointwise.

Our first lemma, which is implicitly in [2], explains why certain results on $l_{\infty}^{2}$ are applicable to the general case.

Lemma 2.1. Let $V$ be a unital bilinear form on $A \times B$. Let $p, q$ be elements such that $0 \leqq p \leqq e_{A}, 0 \leqq q \leqq e_{B}$. Then there is a unital bilinear form $V_{1}$ on $l_{\infty}^{2}$ such that:

$$
\begin{array}{cc}
V_{1}\left(e_{1}, e_{1}\right)=V(p, q), & V_{1}\left(e_{1}, e_{2}\right)=V\left(p, e_{B}-q\right), \\
V_{1}\left(e_{2}, e_{1}\right)=V\left(e_{A}-p, q\right), \quad V_{1}\left(e_{2}, e_{2}\right)=V\left(e_{A}-p, e_{B}-q\right) .
\end{array}
$$

Proof. This follows from the fact that for such $p$,

$$
\left\|\lambda_{1} p+\lambda_{2}\left(e_{A}-p\right)\right\| \leqq \max \left(\left|\lambda_{1}\right|,\left|\lambda_{2}\right|\right)
$$

which is easily seen, e.g. from the Gelfand representation.

Lemma 2.2. Let $V$ be a bilinear form on $l_{\infty}^{2}$ and let $V\left(e_{j}, e_{k}\right)$ be given by the matrix 


$$
\left(\begin{array}{ll}
\alpha & \beta \\
\gamma & \delta
\end{array}\right)
$$

Then $\|V\|=\sup \{|\alpha+\mu \beta|+|\gamma+\mu \delta|:|\mu|=1\}$.

Proof. By convexity and scalar multiplication, $\|V\|$ is the maximum modulus of numbers of the form

$$
\rho V\left(e_{1}+\lambda e_{2}, e_{1}+\mu e_{2}\right)=\rho(\alpha+\mu \beta)+\rho \lambda(\gamma+\mu \delta)
$$

where $|\rho|=|\lambda|=|\mu|=1$. For fixed $\mu$, we can choose $\rho$ and $\lambda$ to make this expression equal to $|\alpha+\mu \beta|+|\gamma+\mu \delta|$.

We shall use this lemma constantly, sometimes with the expression in the equivalent form $\left|\mu^{-1} \alpha+\mu \beta\right|+\left|\mu^{-1} \gamma+\mu \delta\right|$. Clearly, $\beta$ and $\gamma$ can be interchanged, and we may consider $|\mu| \leqq 1$ instead of $|\mu|=1$. Also, if $\bar{V}$ is defined by $\bar{V}\left(e_{j}, e_{k}\right)=\overline{V\left(e_{j}, e_{k}\right)}$, then $\|\bar{V}\|=\|V\|$. The form $W=\frac{1}{2}(V+\bar{V})$ is then hermitian (i.e. $W(x, y)$ is real for real $\left.x, y\right)$ and $\|W\| \leqq\|V\|$.

Lemma 2.3. The matrix of a unital bilinear form on $l_{\infty}^{2}$ is of the form

$$
\left(\begin{array}{ll}
a+i h & b-i h \\
c-i h & d+i h
\end{array}\right)
$$

where each of $a+b, c+d, a+c, b+d, a+d, b+c$ is non-negative and $a+b+c+d=1$.

Proof. Let the matrix be

$$
\left(\begin{array}{ll}
\alpha & \beta \\
\gamma & \delta
\end{array}\right)
$$

Positive functionals $\phi, \psi$ are defined by: $\phi(x)=V(x, e), \psi(y)=V(e, y)$. Hence

$$
\begin{array}{ll}
\phi\left(e_{1}\right)=\alpha+\beta, & \phi\left(e_{2}\right)=\gamma+\delta, \\
\psi\left(e_{1}\right)=\alpha+\gamma, & \psi\left(e_{2}\right)=\beta+\delta
\end{array}
$$

are all real and non-negative. Therefore the matrix has the form stated. Clearly, $V(e, e)=a+b+c+d=1$. Also,

$$
\operatorname{Re} V\left(e_{1}-e_{2}, e_{1}-e_{2}\right)=a-b-c+d \in[-1,1]
$$


so $a+d$ and $b+c$ are non-negative. (From the imaginary part, we see that $|h| \leqq \frac{1}{4}$.)

We shall use the notation of Lemma 2.3 consistently. We give simple direct proofs of the next three results, although they are implied by later theorems.

Lemma 2.4. For a unital form on $l_{\infty}^{2}$ as above, if $a+b=0$, then $a=b=h=0$. Equivalently, if $\phi\left(e_{1}\right)=0$, then $V\left(e_{1}, e_{1}\right)=0$.

Hence if $A, B$ are $C^{*}$-algebras, $V$ is a unital form on $A \times B, 0 \leqq p \leqq e_{A}, 0 \leqq q \leqq e_{B}$ and $\phi(p)=0$, then $V(p, q)=0$.

Proof. Note that $c+d=1$. If $h \neq 0$, then $|c-i h|+|d+i h|>1$, hence $\|V\|>1$. So $h=0$. By Lemma 2.2, we also have $|c+i a|+|d-i a| \leqq 1$, hence $a=0$.

The last statement follows at once, by Lemma 2.1 .

For the real part, it is easy to improve this result, as follows.

Lemma 2.5. For a unital form on $l_{\infty}^{2}$ as above, we have $a^{2} \leqq(a+b)(a+c)$, i.e. $\left|\operatorname{Re} V\left(e_{1}, e_{1}\right)\right|^{2} \leqq \phi\left(e_{1}\right) \psi\left(e_{1}\right)$.

Hence if $V$ is a unital form on $A \times B$, and $0 \leqq p \leqq e_{A}, 0 \leqq q \leqq e_{B}$, then $|\operatorname{Re} V(p, q)|^{2} \leqq$ $\phi(p) \psi(q)$.

Proof. As mentioned above, the form remains unital if we change $h$ to 0 , so we assume that $h=0$. Let

$$
\begin{aligned}
F(\alpha, \beta) & =\operatorname{Re} V\left(e^{i \alpha} e_{1}+e_{2}, e^{i \beta} e_{1}+e_{2}\right) \\
& =a \cos (\alpha+\beta)+b \cos \alpha+c \cos \beta+d
\end{aligned}
$$

Then $F(\alpha, \beta) \leqq 1$ for all $\alpha, \beta$ and $F(0,0)=1$. The second-order partial derivatives at $(0,0)$ are

$$
\begin{aligned}
& \left(D_{11} F\right)(0,0)=-a-b, \\
& \left(D_{22} F\right)(0,0)=-a-c, \\
& \left(D_{12} F\right)(0,0)=-a .
\end{aligned}
$$

The statement follows, by the standard conditions for a local maximum.

Proposition 2.6. For a unital form on $l_{\infty}^{2}$ as above,

$$
b c d+a c d+a b d+a b c \geqq 0 .
$$


Proof. If $a+b=0$, then by Lemma $2.4, a=b=0$, and the expression is zero. Similarly if $c+d=0$. Assume that $a+b>0$ and $c+d>0$. Now

$$
\begin{aligned}
\left|a e^{-i \theta}+b e^{i \theta}\right|^{2} & =a^{2}+2 a b \cos 2 \theta+b^{2} \\
& =a^{2}+2 a b\left(2 x^{2}-1\right)+b^{2},
\end{aligned}
$$

where $x=\cos \theta$. Let

$$
F(x)=\left[a^{2}+2 a b\left(2 x^{2}-1\right)+b^{2}\right]^{1 / 2}+\left[c^{2}+2 c d\left(2 x^{2}-1\right)+d^{2}\right]^{1 / 2} .
$$

Then $F(1)=1$ and $F(x) \leqq 1$ for all $x \in[-1,1]$. Hence $F^{\prime}(1) \geqq 0$, so

$$
\frac{a b}{a+b}+\frac{c d}{c+d} \geqq 0
$$

The statement follows.

Proposition 2.7. Let $V$ be a unital bilinear form on $A \times B$, and let $0 \leqq p \leqq e_{A}, 0 \leqq q \leqq e_{B}$. Then the least possible value for $\operatorname{Re} V(p, q)$ is $-\frac{1}{8}$, and this can occur.

Proof. By Lemma 2.1, we have to show that for a unital form on $l_{\infty}^{2}$, with the above notation, we must have $a \geqq-\frac{1}{8}$. Assume that $a<0$, and write $a=-a^{\prime}$. It is elementary that the form obtained by replacing $b$ and $c$ by $\frac{1}{2}(b+c)$ is still unital: hence we may assume that $b=c$. Since $b \geqq a^{\prime}>0$, Proposition 2.6 becomes $b(a+d)+2 a d \geqq 0$, or

$$
2 a^{\prime} d \leqq b\left(d-a^{\prime}\right)
$$

Now $d \geqq a^{\prime}$ (since $a+d \geqq 0$ ), so in fact $d>a^{\prime}$. Write $d=(1+k) a^{\prime}$. Our inequality becomes

$$
2(1+k) a^{\prime} \leqq k b,
$$

or $b \geqq 2\left(1+k^{-1}\right) a^{\prime}$. Also, $2 b+d-a^{\prime}=a+b+c+d=1$, so

$$
\left(4+\frac{4}{k}+k\right) a^{\prime} \leqq 1
$$

The least value of $k+4 k^{-1}$ is 4 . Hence $a^{\prime} \leqq \frac{1}{8}$.

We now show that a unital form on $l_{\infty}^{2}$ is given by

$$
\left(\begin{array}{rr}
-1 / 8 & 3 / 8 \\
3 / 8 & 3 / 8
\end{array}\right)
$$


so that $a$ can be $-\frac{1}{8}$. (This will follow from Theorem 1, but we give a short direct proof.) We have to show that

$$
\left|-e^{-i \theta}+3 e^{i \theta}\right|+3\left|e^{-i \theta}+e^{i \theta}\right| \leqq 8
$$

for all $\theta$. Writing $x=\cos \theta$, we have

$$
\begin{gathered}
\left|-e^{-i \theta}+3 e^{i \theta}\right|^{2}=10-6 \cos 2 \theta=16-12 x^{2}, \\
\left|e^{-i \theta}+e^{i \theta}\right|^{2}=2+2 \cos 2 \theta=4 x^{2} .
\end{gathered}
$$

The required inequality is: $\left(4-3 x^{2}\right)^{1 / 2}+3 x \leqq 4$ for $|x| \leqq 1$. This is correct, since

$$
(4-3 x)^{2}-\left(4-3 x^{2}\right)=12(x-1)^{2} \geqq 0 .
$$

Remark. The example given is in fact the unique unital form on $l_{\infty}^{2}$ having $a=-\frac{1}{8}$. For, given such a form with $b=c$, we then require $k=2$ in the above proof, hence $d=b=\frac{3}{8}$. We cannot have $b=\frac{3}{8}+\delta, c=\frac{3}{8}-\delta$ with $\delta>0$, since then the expression in Proposition 2.6 is negative. So $b=c=\frac{3}{8}$, and this expression equals 0 ; it will follow from Theorem 1 that $h=0$.

Two further examples may be instructive at this point.

Example 2.8. In the case of real $l_{\infty}^{2}$, it is trivial that

$$
\left(\begin{array}{rr}
-1 / 2 & 1 / 2 \\
1 / 2 & 1 / 2
\end{array}\right)
$$

defines a unital bilinear form. This shows that results 2.4 to 2.7 fail in the real case.

Example 2.9. For any $\lambda$ with $|\lambda|=1$, a unital form on (complex) $l_{\infty}^{2}$ is given by

$$
\frac{1}{4}\left(\begin{array}{ll}
1+\lambda & 1+\lambda \\
1-\lambda & 1-\bar{\lambda}
\end{array}\right)
$$

To show this, let $|\alpha|=1$. Then

$$
\begin{aligned}
& \alpha(1+\lambda)+\bar{\alpha}(1+\bar{\lambda})=2 \operatorname{Re}(\alpha+\alpha \lambda), \\
& \alpha(1-\lambda)+\bar{\alpha}(1-\bar{\lambda})=2 \operatorname{Re}(\alpha-\alpha \lambda),
\end{aligned}
$$

so

$$
|\alpha(1+\lambda)+\bar{\alpha}(1+\bar{\lambda})|+|\alpha(1-\lambda)+\bar{\alpha}(1-\bar{\lambda})| \leqq 4,
$$

since it equals either $4|\operatorname{Re}(\alpha)|$ or $4|\operatorname{Re}(\alpha \lambda)|$. 


\section{Characterization of unital bilinear forms on $\boldsymbol{P}_{\infty}$}

Let $V$ be a unital bilinear form on $l_{\infty}^{2}$ with matrix as above. Haagerup, [2, Lemma 3.2], gives an upper estimate for the imaginary part $h$. We will derive a rather stronger estimate which turns out, together with the other more obvious conditions, to be necessary and sufficient for $V$ to be unital. Our estimate takes the rather unexpected form $h^{2} \leqq E$, where $E$ is the quantity in Proposition 2.6. We see later that this is exactly what is needed to prove our Grothendieck-type theorem.

The proof involves some manipulation, but the principle is elementary. The proof of necessity (which is what is really wanted for applications) essentially consists of squaring twice to remove the square roots implicit in the statement $\|V\| \leqq 1$. The proof of sufficiency then amounts to a careful check that these steps are reversible.

With our usual notation, write

$$
\begin{aligned}
& \Delta=a b-c d, \\
& E=b c d+a c d+a b d+a b c, \\
& F=(a+d)(b+c)(a+c)(b+d) .
\end{aligned}
$$

The next lemma is only needed for sufficiency.

Lemma 3.1. $\Delta^{2}+E=F$.

Proof. Let

$$
G=b c d(b+c+d)+a c d(a+c+d)+a b d(a+b+d)+a b c(a+b+c) .
$$

Then

$$
\begin{aligned}
E & =E(a+b+c+d) \\
& =G+4 a b c d,
\end{aligned}
$$

while

$$
F=G+2 a b c d+a^{2} b^{2}+c^{2} d^{2},
$$

so

$$
F-E=a^{2} b^{2}+c^{2} d^{2}-2 a b c d=\Delta^{2} .
$$

Theorem 1. Let $V$ be the bilinear form on $l_{\infty}^{2}$ with matrix

$$
\left(\begin{array}{ll}
a+i h & b-i h \\
c-i h & d+i h
\end{array}\right)
$$


Then $V$ is unital if and only if the following conditions hold:

(i) $a+b+c+d=1$,

(ii) each of $a+b, c+d, a+c, b+d, a+d, b+c$ is non-negative,

(iii) $h^{2} \leqq b c d+a c d+a b d+a b c(=E)$.

Proof. Observe first that (iii) is equivalent to:

$$
\text { (iiia) }(h \sin \theta+\Delta \cos \theta)^{2} \leqq \Delta^{2}+E \text { for all } \theta \text {. }
$$

Necessity. We have seen (Lemma 2.3) that (i), (ii) are necessary.

With $\theta$ chosen arbitrarily, write

$$
\begin{aligned}
& \sigma=(a+i h) e^{-i \theta}+(b-i h) e^{i \theta} \\
& \tau=(c-i h) e^{-i \theta}+(d+i h) e^{i \theta} .
\end{aligned}
$$

The statement $\|V\|=1$ is equivalent to: $|\sigma|+|\tau| \leqq 1$ for all $\theta$. Now

$$
\begin{aligned}
& |\sigma|^{2}=a^{2}+b^{2}+2 h^{2}+2\left(a b-h^{2}\right) \cos 2 \theta+2 h(a+b) \sin 2 \theta, \\
& |\tau|^{2}=c^{2}+d^{2}+2 h^{2}+2\left(c d-h^{2}\right) \cos 2 \theta-2 h(c+d) \sin 2 \theta
\end{aligned}
$$

hence (recall that $a+b+c+d=1$ ),

$$
|\tau|^{2}-|\sigma|^{2}=c^{2}+d^{2}-a^{2}-b^{2}-2 \Delta \cos 2 \theta-2 h \sin 2 \theta
$$

Provided that we know $|\tau| \leqq 1$ (which is clearly true if $V$ is unital), the inequality $|\sigma| \leqq 1-|\tau|$ is equivalent to $|\sigma|^{2} \leqq(1-|\tau|)^{2}$, hence to

$$
\begin{aligned}
2|\tau| & \leqq 1+|\tau|^{2}-|\sigma|^{2} \\
& =1+c^{2}+d^{2}-a^{2}-b^{2}-2 \Delta \cos 2 \theta-2 h \sin 2 \theta \\
& =1+(c+d)^{2}-(a+b)^{2}+2 \Delta(1-\cos 2 \theta)-2 h \sin 2 \theta \\
& =1+(c+d-a-b)+4 \Delta \sin ^{2} \theta-4 h \sin \theta \cos \theta \\
& =2(c+d)+4 \Delta \sin ^{2} \theta-4 h \sin \theta \cos \theta,
\end{aligned}
$$

(the last two lines again use $a+b+c+d=1$ ). Squaring again, we obtain 


$$
\begin{aligned}
|\tau|^{2} \leqq & (c+d)^{2}+4 \Delta^{2} \sin ^{4} \theta+4 h^{2} \sin ^{2} \theta \cos ^{2} \theta \\
& +4(c+d) \Delta \sin ^{2} \theta-4 h(c+d) \sin \theta \cos \theta-8 h \Delta \sin ^{3} \theta \cos \theta .
\end{aligned}
$$

Writing $\cos 2 \theta=1-2 \sin ^{2} \theta$ in the expression for $|\tau|^{2}$, we see that this is equivalent to

$$
4\left(h^{2}-c d\right) \sin ^{2} \theta \leqq 4 \Delta^{2} \sin ^{4} \theta+4 h^{2} \sin ^{2} \theta \cos ^{2} \theta+4(c+d) \Delta \sin ^{2} \theta-8 h \Delta \sin ^{3} \theta \cos \theta .
$$

For $\sin \theta \neq 0$, this is equivalent to

$$
h^{2}-c d \leqq \Delta^{2} \sin ^{2} \theta+h^{2} \cos ^{2} \theta+(c+d) \Delta-2 h \Delta \sin \theta \cos \theta,
$$

which then holds by continuity also when $\sin \theta=0$. The inequality is equivalent to

$$
\begin{aligned}
h^{2} \sin ^{2} \theta+2 h \Delta \sin \theta \cos \theta & \leqq \Delta^{2} \sin ^{2} \theta+c d+(c+d) \Delta \\
& =\Delta^{2} \sin ^{2} \theta+a b c+a b d+c d(1-c-d) \\
& =\Delta^{2} \sin ^{2} \theta+a b c+a b d+c d(a+b) \\
& =\Delta^{2} \sin ^{2} \theta+E
\end{aligned}
$$

hence to

i.e. statement (iiia).

$$
(h \sin \theta+\Delta \cos \theta)^{2} \leqq \Delta^{2}+E,
$$

Sufficiency. Suppose now that statements (i), (ii), (iiia) hold. Both squaring steps can be reversed provided that the right-hand side in (1) is between 0 and 2 (we will then have $|\tau| \leqq 1$ where needed for the first step). By (iiia) and Lemma 3.1, this amounts to showing that

$$
-1 \leqq c^{2}+d^{2}-a^{2}-b^{2} \pm 2 F^{1 / 2} \leqq 1 .
$$

It is clearly enough to show that $c^{2}+d^{2}+2 F^{1 / 2} \leqq 1$. Since $F=x(1-x) y(1-y)$ for certain $x, y$ in $[0,1]$, we have $F^{1 / 2} \leqq \frac{1}{4}$, so we only need to consider the case where $c^{2}+d^{2}>\frac{1}{2}$. Then one of $c, d$ (say $c$ ) is greater than $\frac{1}{2}$.

Suppose that $a \geqq 0, b \geqq 0$. Since $a+c=1-b-d$ and $x(1-x)$ decreases on the interval $\left[\frac{1}{2}, 1\right]$, we have $(a+c)(b+d) \leqq c(1-c)$. Similarly for $(b+c)(a+d)$. Hence $F^{1 / 2} \leqq c(1-c)$ and

$$
c^{2}+d^{2}+2 F^{1 / 2} \leqq c^{2}+(1-c)^{2}+2 c(1-c)=1 .
$$

Now suppose that $a<0$. Since $a+b \geqq 0$, we have $b+c \geqq c-a>\frac{1}{2}$, hence $(b+c)(a+d) \leqq$ $(c-a)(1-c+a)$, and 


$$
\begin{aligned}
F & \leqq(c-a)(1-c+a)(c+a)(1-c-a) \\
& =\left(c^{2}-a^{2}\right)\left[(1-c)^{2}-a^{2}\right] .
\end{aligned}
$$

So $F^{1 / 2} \leqq c(1-c)$ and the conclusion follows as before.

Corollary 3.2 ([2, Lemma 3.2]). With the above notation, $h^{2} \leqq F$ and also

$$
\begin{aligned}
h^{2} & \leqq(a+b)(c+d)(a+c)(b+d) \\
& =\left[\phi\left(e_{1}\right)-\phi\left(e_{1}\right)^{2}\right]\left[\psi\left(e_{1}\right)-\psi\left(e_{1}\right)^{2}\right] .
\end{aligned}
$$

Proof. By Lemma 3.1 (for the second statement, interchange $b$ and $d$ ).

Note. By considering scalar multiples, we see that the statement $\|V\|=V(e, e)(\neq 1)$ is equivalent to (ii) and $h^{2}(a+b+c+d) \leqq E$.

\section{Grothendieck-type theorems for $P_{\infty}^{2}$}

First we consider linear operators into a Hilbert space. Let $A$ be a commutative $C^{*}$-algebra, $H$ a Hilbert space and $T$ an operator from $A$ to $H$ with $\|T\|=\left\|T\left(e_{A}\right)\right\|=1$. By [5, Theorem 9.4], we then have $\|T x\|^{2} \leqq 2 f\left(|x|^{2}\right)$ for all $x \in A$, where $f(x)=$ $\left\langle T x, T e_{A}\right\rangle$. This shows that $\pi_{2}(T) \leqq \sqrt{2}\|T\|$ (where $\pi_{2}$ denotes 2 -summing norm), and that for operators of this kind, unlike bilinear forms, there is an automatic formula for the dominating functional, if we disregard the fact that the factor 2 is not optimal (in fact, $\pi_{2}(T) \leqq(2 / \sqrt{\pi})\|T\|$, so by Pietsch's theorem, there is a functional $g$ such that $\|T x\|^{2} \leqq(4 / \pi) g\left(|x|^{2}\right)$; see e.g. [3, Theorems 5.2 and 9.11]). We now describe a case where the 2 can be replaced by 1 . We denote by $L(X, Y)$ the space of continuous linear operators from $X$ to $Y$.

Lemma 4.1. (Cf. [3, exercise on p. 103]). Let $T \in\left(L\left(l_{\infty}^{n}, H\right)\right.$ be such that $\left\langle T e_{j}, T e_{k}\right\rangle$ is real and non-negative for all $j, k$. Then a positive linear functional $f$ is defined by: $f(x)=\langle T x, T e\rangle$ and

$$
\|T x\|^{2} \leqq f\left(|x|^{2}\right)
$$

for all $x$. (Consequently $\pi_{2}(T)=\|T\|$.)

Proof. Write $x=\sum_{j=1}^{n} x(j) e_{j}$ and $T e_{j}=y_{j}$. Then

$$
\|T x\|^{2}=\sum_{j} \sum_{k} x(j) \overline{x(k)}\left\langle y_{j}, y_{k}\right\rangle
$$




$$
f\left(|x|^{2}\right)=\sum_{j} \sum_{k} x(j) \overline{x(j)}\left\langle y_{j}, y_{k}\right\rangle
$$

Since $\left\langle y_{k}, y_{j}\right\rangle=\left\langle y_{j}, y_{k}\right\rangle$, we have

$$
\begin{aligned}
f\left(|x|^{2}\right)-\|T x\|^{2} & =\sum_{j<k}[x(j) \overline{x(j)}+x(k) \overline{x(k)}-x(j) \overline{x(k)}-\overline{x(j)} x(k)]\left\langle y_{j}, y_{k}\right\rangle \\
& =\sum_{j<k}|x(j)-x(k)|^{2}\left\langle y_{j}, y_{k}\right\rangle \\
& \geqq 0
\end{aligned}
$$

Since $\|T\|=\|T e\|$ and $\|f\|=f(e)=\|T\|^{2}$, it follows that $f$ is positive and $\pi_{2}(T)=\|T\|$.

The condition in Lemma 4.1 implies that $\|T\|=\|T e\|$. The special feature of the case $n=2$ is that the converse applies, as we now show.

Proposition 4.2. (i) Let $T \in L\left(l_{\infty}^{2}, H\right)$ satisfy $\|T\|=\|T e\|$, and let $f(x)=\langle T x, T e\rangle$. Then for all $x$ in $l_{\infty}^{2}$,

$$
\|T x\|^{2} \leqq f\left(|x|^{2}\right)
$$

(ii) For any $T \in L\left(l_{\infty}^{2}, H\right)$, we have $\pi_{2}(T)=\|T\|$.

Proof. (i) Let $T e_{j}=y_{j}$. By Lemma 4.1, we only need to show that $\left\langle y_{1}, y_{2}\right\rangle \geqq 0$. By hypothesis, $\left\|y_{1}+y_{2}\right\| \geqq\left\|y_{1}+\alpha y_{2}\right\|$, hence $\operatorname{Re}\left\langle y_{1}, y_{2}\right\rangle \geqq \operatorname{Re}\left\langle y_{1}, \alpha y_{2}\right\rangle$, for all $\alpha$ with $|\alpha|=1$. Choose $\alpha$ so that $\left\langle y_{1}, \alpha y_{2}\right\rangle=\left|\left\langle y_{1}, y_{2}\right\rangle\right|$. Then we clearly have $\left\langle y_{1}, y_{2}\right\rangle=\left|\left\langle y_{1}, y_{2}\right\rangle\right|$.

(ii) For $T$ satisfying $\|T\|=\|T e\|$, the statement follows from (i). For other $T$, choose an element $x_{0}$ such that $\left|x_{0}\right|=e$ and $\left\|T x_{0}\right\|=\|T\|$, and apply (i) to the operator $T_{1}$ defined by $T_{1}(x)=T\left(x x_{0}\right)$.

Theorem 2. (i) Let $V$ be a unital bilinear form on $l_{\infty}^{2}$. Let $\phi(x)=V(x, e), \psi(y)=V(e, y)$. Then for all $x, y$ in $l_{\infty}^{2}$,

$$
|V(x, y)|^{2} \leqq \phi\left(|x|^{2}\right) \psi\left(|y|^{2}\right)
$$

(ii) Let $V$ be any bilinear form on $l_{\infty}^{2}$ with $\|V\|=1$. Then there are states $\phi, \psi$ such that (1) holds. In other words, the $2 \times 2$ Grothendieck constant equals 1 .

Proof. (i) implies (ii) (standard). There exist elements $x_{0}, y_{0}$ such that $\left|x_{0}\right|=\left|y_{0}\right|=e$ and $V\left(x_{0}, y_{0}\right)=1$. Define $V_{1}$ by

$$
V_{1}(x, y)=V\left(x x_{0}, y y_{0}\right)
$$


Then $V_{1}$ is unital, so by (i) there exist states $\phi, \psi$ such that for all $x, y$ :

$$
\left|V_{1}(x, y)\right|^{2} \leqq \phi\left(|x|^{2}\right) \psi\left(|y|^{2}\right)
$$

Since $V(x, y)=V_{1}\left(x_{0}^{-1} x, y_{0}^{-1} y\right)$ and $\left|x_{0}^{-1} x\right|=|x|$ (etc.), the same inequality is satisfied by $V(x, y)$.

Proof of (i). First, consider the case where one of $\phi\left(e_{1}\right), \phi\left(e_{2}\right)\left(\right.$ say $\left.\phi\left(e_{2}\right)\right)$ is 0 . By Lemma 2.4, we then have (with our previous notation) $c=d=h=0$ and $a+b=1$. It follows easily that $V(x, y)=\phi(x) \psi(y)$, and the statement follows since $|\phi(x)|^{2} \leqq \phi\left(|x|^{2}\right)$, etc.

Suppose now that $\phi\left(e_{1}\right)>0, \phi\left(e_{2}\right)>0$. Then an inner product is defined on $\mathbb{C}^{2}$ by

$$
\langle x, y\rangle_{\phi}=\phi(x \bar{y})
$$

Let $H_{\phi}$ be $\mathbb{C}^{2}$ equipped with this inner product. For any $x$,

$$
\begin{aligned}
V\left(x, e_{k}\right) & =\sum_{j=1}^{2} x(j) V\left(e_{j}, e_{k}\right) \\
& =\phi\left(x z_{k}\right)
\end{aligned}
$$

where

$$
z_{k}(j)=\frac{V\left(e_{j}, e_{k}\right)}{\phi\left(e_{j}\right)} \quad(j=1,2)
$$

Define a linear operator $T$ from $l_{\infty}^{2}$ to $H_{\phi}$ by: $T e_{k}=z_{k}$ for $k=1,2$. Then the above shows that

$$
V(x, y)=\phi(x \cdot T y)
$$

for all $x, y$. Note that (1) $T e=z_{1}+z_{2}=e$, (2) $\phi(T y)=V(e, y)=\psi(y)$. Now with our previous notation,

$$
\begin{aligned}
\left\langle z_{1}, z_{2}\right\rangle_{\phi} & =\sum_{j=1}^{2} \phi\left(e_{j}\right) z_{1}(j) \overline{z_{2}(j)} \\
& =\frac{(a+i h)(b+i h)}{a+b}+\frac{(c-i h)(d-i h)}{c+d} \\
& =\frac{a b-h^{2}}{a+b}+\frac{c d-h^{2}}{c+d}
\end{aligned}
$$

By Theorem 1, this is non-negative. By Lemma 4.1, $\|T y\|_{\phi}^{2} \leqq f\left(|y|^{2}\right)$ for all $y$, where 


$$
\begin{aligned}
f(y) & =\langle T y, T e\rangle_{\phi} \\
& =\phi(T y) \quad \text { by (1) above) } \\
& =\psi(y) \quad \text { by }(2)) .
\end{aligned}
$$

So

$$
\begin{aligned}
|V(x, y)|^{2} & =\left|\langle T y, \bar{x}\rangle_{\phi}\right|^{2} \\
& \leqq\|x\|_{\phi}^{2}\|T y\|_{\phi}^{2} \\
& \leqq \phi\left(|x|^{2}\right) \psi\left(|y|^{2}\right) .
\end{aligned}
$$

Corollary 4.3. Let $A, B$ be $C^{*}$-algebras, $V$ a unital bilinear form on $A \times B$. If $0 \leqq x \leqq e_{A}$, $0 \leqq y \leqq e_{B}$, then

$$
|V(x, y)|^{2} \leqq \phi(x) \psi(y)
$$

(where $\phi, \psi$ are defined as before).

Proof. For $l_{\infty}^{2}$, this follows immediately, since $\phi\left(x^{2}\right) \leqq \phi(x)$, etc. The result for general $C^{*}$-algebras follows, by Lemma 2.1 .

This corollary combines Lemmas 3.1 and 3.2 of [2] (where the real and imaginary parts are considered separately) in the form actually required for the proof of the generalized Grothendieck inequality. We remark that it is quite easy to deduce Corollary 4.3 from Theorem 1 without Theorem 2: in our usual notation, one shows that $a^{2}+h^{2} \leqq(a+b)(a+c)$. Since even the proof of our Theorem 1 is as much work as the proof of Haagerup's lemmas, we give an alternative short proof of Corollary 4.3 in Section 5 .

\section{Higher dimensions; Grothendieck's inequality}

The standard Grothendieck inequality states that for a bilinear form $V$ on $l_{\infty}^{n}$ with norm 1 , there are states $\phi^{\prime}, \psi^{\prime}$ such that $|V(x, y)|^{2} \leqq K_{G} \phi^{\prime}\left(|x|^{2}\right) \psi^{\prime}\left(|y|^{2}\right)$ for all $x, y$. One approach to this is somewhat analogous to our proof of Theorem 2 (cf. [5, chapter 9]; $\left[3\right.$, p. 115]). Once one has found $\phi^{\prime}$ such that $|V(x, y)| \leqq K\|x\|_{\phi^{\prime}}\|y\|_{\infty}$, Theorem 9.4 of [5] does the rest. The hard part, proving the existence of $\phi^{\prime}$, is equivalent to showing that $\pi_{2}(S) \leqq K\|S\|$, where $S: l_{\infty}^{n} \rightarrow l_{1}^{n}$ is given by $(S x)(y)=V(x, y)$. For $n>2$, our $\phi$ will not serve as $\phi^{\prime}$. What can be said about our $\phi, \psi$ is the following. Let $V$ be a unital form on $l_{\infty}^{n}$. By Corollary 4.3 , for each $j, k$,

$$
\left|V\left(e_{j}, e_{k}\right)\right|^{2} \leqq \phi\left(e_{j}\right) \psi\left(e_{k}\right)
$$


For elements $x, y$, we have

$$
V(x, y)=\sum_{j=1}^{n} \sum_{k=1}^{n} x(j) V\left(e_{j}, e_{k}\right) y(k)
$$

and hence

$$
\begin{aligned}
|V(x, y)|^{2} & \leqq n^{2} \sum_{j=1}^{n} \sum_{k=1}^{n}|x(j)|^{2}|y(k)|^{2} \phi\left(e_{j}\right) \psi\left(e_{k}\right) \\
& =n^{2} \phi\left(|x|^{2}\right) \psi\left(|y|^{2}\right) .
\end{aligned}
$$

Example 3.8 in [2], exhibits a unital form on $l_{\infty}^{2} \times L_{\infty}(I)$, where $I=[-\pi / 2, \pi / 2]$, for which there is no constant $C$ such that

$$
|V(x, y)|^{2} \leqq C^{2} \phi\left(|x|^{2}\right) \psi\left(|y|^{2}\right)
$$

for all $x, y$. By finite approximation, it follows that the $n^{2}$ appearing above cannot be replaced by a constant independent of $n$.

Example 4.4. The following example (which is adopted from Haagerup's) shows that even in the case of $l_{\infty}^{2} \times l_{\infty}^{3}$, the best value of $C$ in the above statement is at least $\sqrt{2}$, and that $\phi, \psi$ is not the best choice of dominating functionals. This demonstrates a fundamental difference between the $2 \times 2$ and $2 \times 3$ cases, rather analogous to the difference between the $C^{*}$-algebras on $Z_{2} * Z_{2}$ and $Z_{2} * Z_{3}$ (cf. [6]).

Let $I=[-\pi / 2, \pi / 2]$ and let $V_{0}$ be Haagerup's bilinear form:

$$
V_{0}(x, f)=\frac{1}{4} x(1) \int_{I} f(t) e^{i t} d t+\frac{1}{4} x(2) \int_{I} f(t) e^{-i t} d t
$$

Choose $\delta>0$ and partition $I$ into

$$
I_{1}=\left[\frac{\pi}{2}-\delta, \frac{\pi}{2}\right]
$$

$I_{2}=-I_{1}$ and $I_{3}=I \backslash\left(I_{1} \cup I_{2}\right)$. Let $f_{j}$ be the characteristic function of $I_{j}$. A unital form $V$ is defined on $l_{\infty}^{2} \times l_{\infty}^{3}$ by putting $V\left(e_{j}, e_{k}\right)=V_{0}\left(e_{j}, f_{k}\right)$. The matrix $\left.V\left(e_{j}, e_{k}\right)\right)$ is

$$
\frac{1}{4}\left(\begin{array}{ccc}
1-e^{-i \delta} & 1-e^{i \delta} & 2 \cos \delta \\
1-e^{i \delta} & 1-e^{-i \delta} & 2 \cos \delta
\end{array}\right)
$$

and we have 


$$
\begin{aligned}
& \phi(x)=\frac{1}{2} x(1)+\frac{1}{2} x(2), \\
& \psi(y)=\frac{1}{2}[y(1)+y(2)](1-\cos \delta)+y(3) \cos \delta .
\end{aligned}
$$

Let $x_{0}=(1,-1), y_{0}=(1,-1,0)$. Then $V\left(x_{0}, y_{0}\right)=i \sin \delta$, while $\psi\left(y_{0}^{2}\right)=1-\cos \delta$. The statement follows, since $\sin ^{2} \delta /(1-\cos \delta) \rightarrow 2$ as $\delta \rightarrow 0$.

We now show how a better choice of dominating functionals can be made. For $u, v$ in $l_{2}^{2}$, write $[u, v]=u(1) v(1)+u(2) v(2)$. Then $V(x, y)=[S x, T y]$, where $S x=x / \sqrt{2}$ and $T$ is given by the matrix for $V$ multiplied by $\sqrt{2}$. With $\phi$ as before, we have $\|S x\|^{2}=\phi\left(|x|^{2}\right)$. We shall define a positive functional $\psi_{1}$, with norm tending to 1 as $\delta \rightarrow 0$, such that $\|T y\|^{2} \leqq \psi_{1}\left(|y|^{2}\right)$ for all $y$, hence such that $|V(x, y)|^{2} \leqq \phi\left(|x|^{2}\right) \psi_{1}\left(\left.|y|\right|^{2}\right)$ for all $x, y$. Now

$$
\left\langle T e_{1}, T e_{3}\right\rangle=\left\langle T e_{2}, T e_{3}\right\rangle=-\left\langle T e_{1}, T e_{2}\right\rangle=r(\delta),
$$

where $r(\delta)=\frac{1}{2} \cos \delta(1-\cos \delta)$. Since $T y=\sum_{j=1}^{3} y(j)\left(T e_{j}\right)$, we have

$$
\begin{aligned}
\|T y\|^{2} & =\sum_{j=1}^{3}|y(j)|^{2}\left\|T e_{j}\right\|^{2}+2 \operatorname{Re}[y(2) \overline{y(3)}+y(3) \overline{y(1)}-y(1) \overline{y(2)}] r(\delta) \\
& \leqq \frac{1}{2}\left(|y(1)|^{2}+|y(2)|^{2}\right)(1-\cos \delta)+|y(3)|^{2} \cos ^{2} \delta+2 \sum_{j=1}^{3}|y(j)|^{2} r(\delta) \\
& =\frac{1}{2}\left(|y(1)|^{2}+|y(2)|^{2}\right)\left(1-\cos ^{2} \delta\right)+|y(3)|^{2} \cos \delta \\
& =\psi_{1}\left(|y|^{2}\right)
\end{aligned}
$$

where $\left\|\psi_{1}\right\|=1-\cos ^{2} \delta+\cos \delta$. (The difference between $\psi$ and $\psi_{1}$ is that $1-\cos \delta$ has been replaced by $1-\cos ^{2} \delta$.)

\section{Two short proofs}

\section{Proof of Haagerup's Lemma 3.2 (our Corollary 4.3 with an extra constant)}

The following is in the spirit of Haagerup's proof, but considerably shorter. With our previous notation, write $a+b=\phi, a+c=\psi, a+i h=A$. We assume our Lemma 2.5 (or Haagerup's 3.1), giving $a^{2} \leqq \phi \psi$. We will show that $|A|^{2} \leqq 2 \phi \psi$ (i.e. Corollary 4.3 with an extra factor of 2). Clearly, this holds if $|a| \geqq|h|$, so we assume that $|a| \leqq|h|$ and also w.l.o.g. $h \geqq 0$. Then $A=r e^{i \theta}$, where $\pi / 4 \leqq \theta \leqq 3 \pi / 4$ (the argument only needs $0 \leqq \theta \leqq 3 \pi / 4$ ).

If $\phi=0$, the result is immediate, by Lemma 2.4 , so we assume that $\phi>0$ and (again w.l.o.g.) that $\phi \leqq \psi$. Let $\rho^{2}=\phi / \psi$, so that $\rho \psi=(\phi \psi)^{1 / 2}$.

Let $x=(\lambda, 1), y=(\mu, 1)$, where $|\lambda|=|\mu|=1$. Writing $x$ as $e-(1-\lambda) e_{1}$ (etc.), one sees that

$$
V(x, y)=1-(1-\lambda) \phi-(1-\mu) \psi+(1-\lambda)(1-\mu) A .
$$


Let $\lambda=e^{i \alpha}, \mu=e^{i \beta}$. Then the statement $\operatorname{Re} V(x, y) \leqq 1$ says

$$
\operatorname{Re}[(1-\lambda)(1-\mu) A] \leqq(1-\cos \alpha) \phi+(1-\cos \beta) \psi
$$

Now choose $\mu=e^{i \beta}$ so that $0 \leqq \beta \leqq \pi / 2$ and $1-\cos \beta=\rho^{2}$. Then $\cos (\theta+\beta) \leqq \cos \theta$ (a diagram is helpful!), hence $\operatorname{Re}(\mu A) \leqq \operatorname{Re}(A)$, or $\operatorname{Re}(B) \geqq 0$, where $B=(1-\mu) A$. It is now elementary that for $\lambda$ equal to either $i$ or $-i$, we have $\operatorname{Re}(1-\lambda) B \geqq|B|$.

Now $|1-\mu|^{2}=2-2 \cos \beta=2 \rho^{2}$, hence $|B|=\sqrt{2} \rho|A|$. Form (1), we now have

$$
\sqrt{2} \rho|A| \leqq \phi+(1-\cos \beta) \psi=2 \rho^{2} \psi
$$

hence $|A| \leqq \sqrt{2} \rho \psi=(2 \phi \psi)^{1 / 2}$.

\section{Proof that the $2 \times 2$ Grothendieck constant is 1}

The following is more direct (though weaker) than our Theorem 2, or the proofs in [1], [7].

Let $V$ be a bilinear form (not necessarily unital) on $l_{\infty}^{2}$ with $\|V\|=1$. We may assume that its matrix has the form

$$
\left(\begin{array}{cc}
a_{1} & \rho a_{2} \\
b_{1} & \sigma \rho b_{2}
\end{array}\right)
$$

where $a_{1}, a_{2}, b_{1}, b_{2} \geqq 0$ and $|\rho|=|\sigma|=1$ (our earlier notation is no longer in force!). For any $\alpha$ with $|\alpha|=1$, we have

$$
\left|a_{1}+\alpha a_{2}\right|+\left|b_{1}+\alpha \sigma b_{2}\right| \leqq 1 .
$$

Let $x_{1}, x_{2}$ be elements of a Hilbert space with $\left\|x_{1}\right\|=\left\|x_{2}\right\|=1$. Let

$$
y=a_{1} x_{1}+\rho a_{2} x_{2}, \quad z=b_{1} x_{1}+\sigma \rho b_{2} x_{2} .
$$

By the Lindenstrauss-Pelczyński form of Grothendieck's inequality, we have to show that $\|y\|+\|z\| \leqq 1$. We will show in fact that we can choose $\alpha$ with $|\alpha|=1$ to satisfy

$$
\begin{gathered}
\|y\| \leqq\left|a_{1}+\alpha a_{2}\right|, \\
\|z\| \leqq\left|b_{1}+\alpha \sigma b_{2}\right| .
\end{gathered}
$$

Let $\left\langle x_{1}, \rho x_{2}\right\rangle=\beta$. Suppose that $|\alpha|=|\delta|=1$ and $\operatorname{Re} \alpha \geqq \operatorname{Re}(\beta \bar{\delta})$. Then for any $c_{1}, c_{2} \geqq 0$,

$$
\left\|c_{1} x_{1}+\delta \rho c_{2} x_{2}\right\|^{2}=c_{1}^{2}+c_{2}^{2}+c_{1} c_{2}(\beta \bar{\delta}+\bar{\beta} \delta)
$$




$$
\begin{aligned}
& \leqq c_{1}^{2}+c_{2}^{2}+c_{1} c_{2}(\alpha+\bar{\alpha}) \\
& =\left|c_{1}+\alpha c_{2}\right|^{2} .
\end{aligned}
$$

Hence (2), (3) will be satisfied if $\operatorname{Re} \alpha \geqq \operatorname{Re} \beta$ and $\operatorname{Re}(\alpha \sigma) \geqq \operatorname{Re}(\beta \bar{\sigma})$. Let $\beta=r e^{i \theta}, \sigma=e^{i \phi}$ (where $-\pi \leqq \phi \leqq \pi)$. If $r \neq 0$, let $\alpha=e^{i \theta}$ : then the conditions are satisfied, since $\operatorname{Re}(\alpha \sigma)=$ $\cos (\theta-\phi)$ and $\operatorname{Re}(\beta \bar{\sigma})=r \cos (\theta-\phi)$. If $r=0$, let $\alpha=e^{i \phi / 2}$.

\section{REFERENCES}

1. A. M. DAvIE, Matrix norms related to Grothendieck's inequality, in Banach Spaces (ed. N. Kalton and E. Saab, Lecture Notes in Math. 1166, Springer, Berlin 1985), 22-26.

2. U. HaAgeruP, The Grothendieck inequality for bilinear forms on $C^{*}$-algebras, Adv. Math. 56 (1985), 93-116.

3. G. J. O. Jameson, Summing and Nuclear Norms in Banach Space Theory (London Math. Soc. Student Texts 8, Cambridge Univ. Press, 1987).

4. G. PISIER, Grothendieck's theorem for non-commutative $C^{*}$-algebras with an appendix on Grothendieck's constant. J. Funct. Anal. 29 (1978), 397-415.

5. G. Pisier, Factorization of Linear Operators and Geometry of Banach Spaces (American Math. Soc., Providence 1986).

6. I. Raeburn and A. M. Sinclair, The $C^{*}$-algebra generated by two projections, Math. Scand 65 (1989), 278-290.

7. A. Tonge, The complex Grothendieck inequality for $2 \times 2$ matrices, Bull. Greek Math. Soc. 27 (1986), 133-135.

Department of Mathematics

LANCASTER UNIVERSITY

LANCASTER LA1 4YF 